\title{
NÓDULOS COM SINAL DO HALO NA ASPERGILOSE PULMONAR ANGIOINVASIVA: CORRELAÇÃO DA TOMOGRAFIA COMPUTADORIZADA DE ALTA RESOLUÇÃO COM A ANATOMOPATOLOGIA*
}

\author{
Edson Marchiori ${ }^{1}$, Paulo Marcos Valiante ${ }^{2}$, Arthur Soares Souza Jr. ${ }^{3}$
}

Resumo O objetivo do trabalho é apresentar os achados precoces da aspergilose angioinvasiva na tomografia computadorizada de alta resolução e correlacioná-los com a anatomopatologia. Foram analisadas as tomografias computadorizadas de alta resolução de dois pacientes com aspergilose angioinvasiva, ambos com leucemia, e feita a correlação com os achados de necropsia. Os nódulos corresponderam a infartos hemorrágicos, com necrose de coagulação, permeados por hifas dos fungos. $O$ halo em vidro fosco correspondeu a hemorragia intra-alveolar. Os nódulos com sinal do halo observados na tomografia computadorizada de alta resolução de pacientes com aspergilose angioinvasiva apresentaram estreita correlação com os aspectos anatomopatológicos. As alterações dos nódulos com sinal do halo demonstradas à anatomia patológica explicam claramente as características da lesão à tomografia computadorizada de alta resolução.

Unitermos: Aspergilose angioinvasiva. Tomografia computadorizada de alta resolução. Anatomopatologia. Pulmões.

Abstract Halo sign nodules in angioinvasive pulmonary aspergillosis: correlation of high-resolution computed tomog-
raphy findings with anatomopathology.
The aim of this study was to correlate the early findings observed on high-resolution computed tomography
with anatomopathology results of patients with angioinvasive aspergillosis. The authors studied two pa-
tients with leukemia. The nodules seen on high-resolution computed tomography corresponded to areas of
hemorrhagic infarction, necrotic coagulation and fungal hyphae infiltration whereas ground glass halo cor-
responded to intraalveolar hemorrhage. Nodules with halo sign were closely correlated with anatomopathology
findings. We concluded that the anatomopathology findings can clearly explain the image features of the
lesions observed on high-resolution computed tomography.
Key words: Angioinvasive aspergillosis. High-resolution computed tomography. Anatomopathology. Lungs.

\section{INTRODUÇÃO}

As alterações pulmonares causadas pelo Aspergillus são variadas e dependentes do estado imunológico e das condições pulmonares do paciente. Reação de hipersensibilidade à inalação de esporos pode causar alveolite alérgica aguda, e colonização

* Trabalho realizado no Departamento de Radiologia da Universidade Federal Fluminense (UFF), Niterói, RJ, e nos Serviços de Radiodiagnóstico e de Anatomia Patológica do Hospital Universitário Clementino Fraga Filho da Universidade Federal do Rio de Janeiro (HUCFF-UFRJ), Rio de Janeiro, RJ.

1. Professor Titular de Radiologia da UFF, Coordenador Adjunto do Curso de Pós-graduação em Radiologia da UFRJ.

2. Professor Assistente do Departamento de Patologia da UFRJ.

3. Professor Adjunto de Radiologia da Faculdade de Medicina de São José do Rio Preto (Famerp), Membro do Instituto de Radiodiagnóstico Rio Preto (Ultra-X), São José do Rio Preto, SP.

Endereço para correspondência: Prof. Dr. Edson Marchiori. Rua Thomaz Cameron, 438, Valparaíso. Petrópolis, RJ, 25685-120. E-mail: edmarchiori@zipmail.com.br

Recebido para publicação em 20/3/2002. Aceito, após revisão, em 28/3/2002. brônquica pode causar aspergilose broncopulmonar alérgica. Esta última é encontrada especialmente em pacientes com asma ou fibrose cística. Micetomas se desenvolvem por colonização secundária de cavidades pulmonares pré-existentes. Aspergilose invasiva e semi-invasiva afetam particularmente pacientes com estado imunológico alterado ${ }^{(\mathbf{1})}$.

A aspergilose pulmonar invasiva é uma complicação grave e freqüente em pacientes imunocomprometidos. As condições predisponentes incluem leucemia aguda com neutropenia, uso de corticosteróides e outros agentes imunossupressivos, transplantes de órgãos e linfomas ${ }^{(2)}$.

O reconhecimento precoce da aspergilose angioinvasiva (AAI), com a adoção de tratamento agressivo, com terapia fúngica em altas doses, é imperativo nestes pacientes, elevando a sua sobrevida ${ }^{(2-4)}$.

A tomografia computadorizada de alta resolução (TCAR) é um método não-inva- sivo, podendo ter utilidade no diagnóstico precoce da doença, especialmente detectando nódulos com o sinal do halo, numa fase em que os achados na radiologia convencional são inespecíficos, as culturas fúngicas são negativas e os procedimentos por biópsia estão contra-indicados, por causa da trombocitopenia ${ }^{(3,4)}$.

Este estudo se propõe a apresentar dois casos de AAI caracterizados pela presença de nódulos com o sinal do halo nas TCAR, e fazer a correlação com os achados anatomopatológicos.

\section{CASUÍSTICA E MÉTODOS}

Neste trabalho foram avaliadas as alterações tomográficas observadas em dois pacientes com AAI em fase precoce, um deles do sexo masculino, de 35 anos de idade, e o outro do sexo feminino, de 30 anos de idade, ambos com leucemia aguda, acompanhados no Hospital Universitário 
Clementino Fraga Filho da Universidade Federal do Rio de Janeiro, e feita a correlação com achados de necropsia. Os exames foram feitos num tomógrafo Somaton DR (Siemens), tendo sido usado, em ambos, o protocolo de alta resolução, com cortes axiais de $2 \mathrm{~mm}$ de espessura e $10 \mathrm{~mm}$ de incremento, dos ápices até as bases pulmonares. O material anatomopatológico foi analisado com colorações pela hematoxilina-eosina e prata metenamina (Grocott).

\section{RESULTADOS}

Nos dois casos foi observada a presença de nódulo único, de limites mal definidos, circundado por halo de atenuação em vidro fosco, um deles no lobo superior do pulmão direito e o outro no lobo inferior esquerdo. Um dos pacientes apresentava também consolidações esparsas nas bases pulmonares e derrame pleural bilateral.

Na correlação com a anatomopatologia observou-se que o centro dos nódulos correspondia a área de necrose de coagulação, com hifas do fungo de permeio, dispostas paralelamente, irradiando-se do centro para a periferia. Angioinvasão foi também detectada nos dois casos. O halo em vidro fosco correspondeu a hemorragia ocupando os espaços alveolares perinodulares.

\section{DISCUSSÃO}

As formas invasivas e semi-invasivas da aspergilose acometem basicamente pacientes com algum tipo de imunodepressão.

A aspergilose semi-invasiva ocorre em pacientes levemente imunossuprimidos, com baixa da imunidade causada por doen- ças debilitantes crônicas, com alteração nos mecanismos de defesa (malnutrição, alcoolismo, diabetes, etc.) ou em prolongada terapia com corticosteróides. Esta forma granulomatosa crônica da infecção é também conhecida como aspergilose pulmonar necrotizante crônica. Ela lembra muitas doenças crônicas, como tuberculose, actinomicose ou histoplasmose. Os aspectos radiológicos incluem áreas de consolidação focal ou lesões com aspecto de massa e evolução arrastada, freqüentemente com espessamento pleural associado. Este quadro pode ser indistinguível da tuberculose ${ }^{(\mathbf{5 , 6})}$.

A aspergilose invasiva ocorre quase exclusivamente em pacientes imunocomprometidos, com neutropenia grave, sendo uma complicação relativamente comum nestes pacientes ${ }^{(7,8)}$. Por várias razões, contudo, tem havido um importante aumento

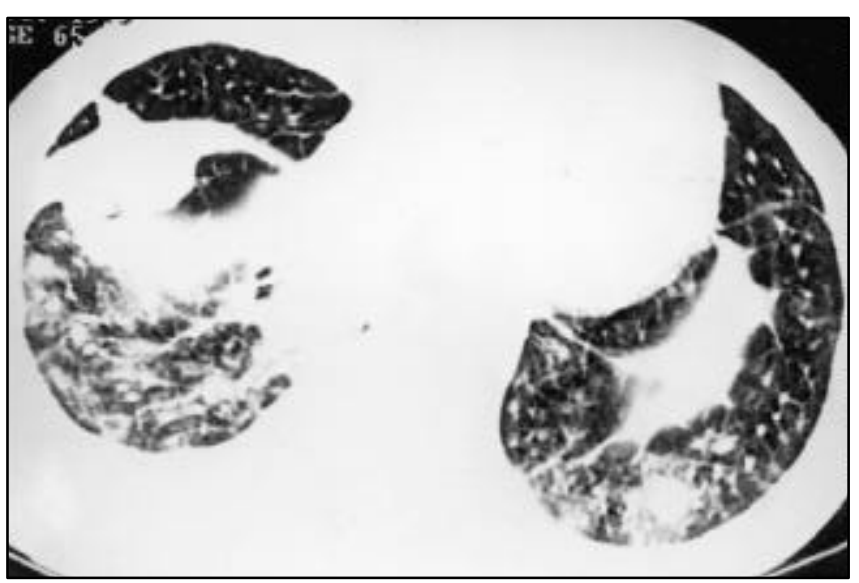

A

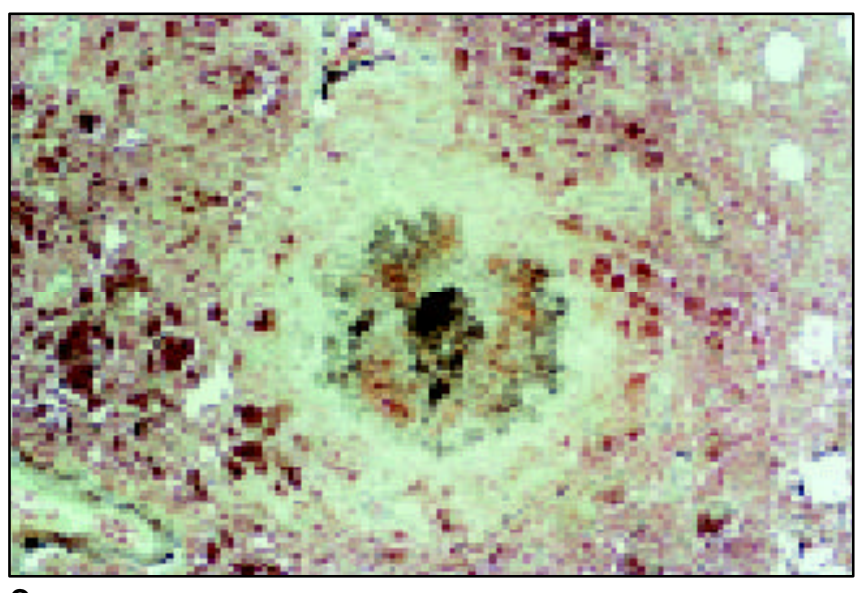

c

Figura 1. Em A, TCAR mostrando nódulo circundado por halo em vidro fosco no lobo inferior esquerdo e outros nódulos menores em ambas as bases pulmonares. Observar também áreas de consolidação esparsas, e derrame pleural, mais evidente à direita. Em B, coloração pela hematoxilina-eosina, observa-se um dos nódulos menores, com o centro necrótico envolvido por um infiltrado leucocitário e, mais externamente, por espaços alveolares cheios de sangue. Em C, coloração pela prata, evidencia-se, em negro, as hifas do Aspergillus no interior do nódulo, num arranjo radial, do centro para a periferia. Em D, em maior aumento, destaca-se melhor o aspecto das hifas na periferia do nódulo.

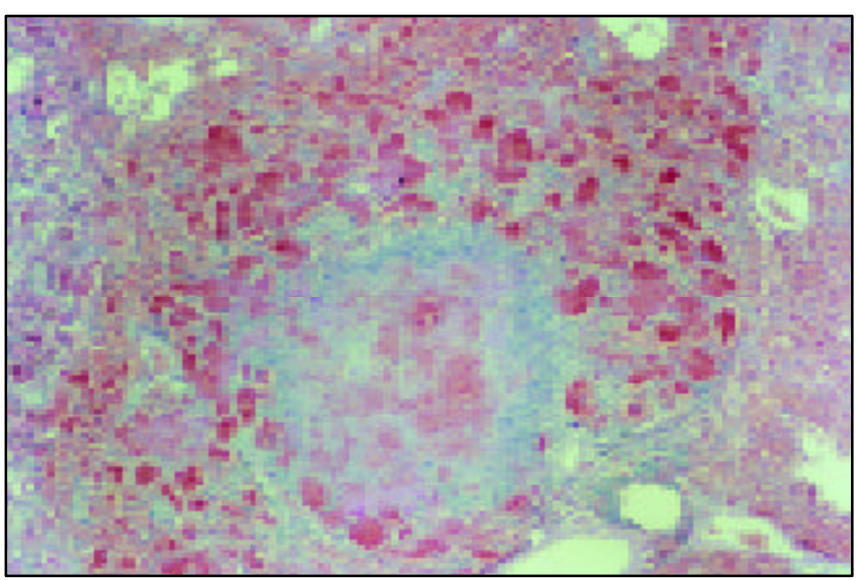

B

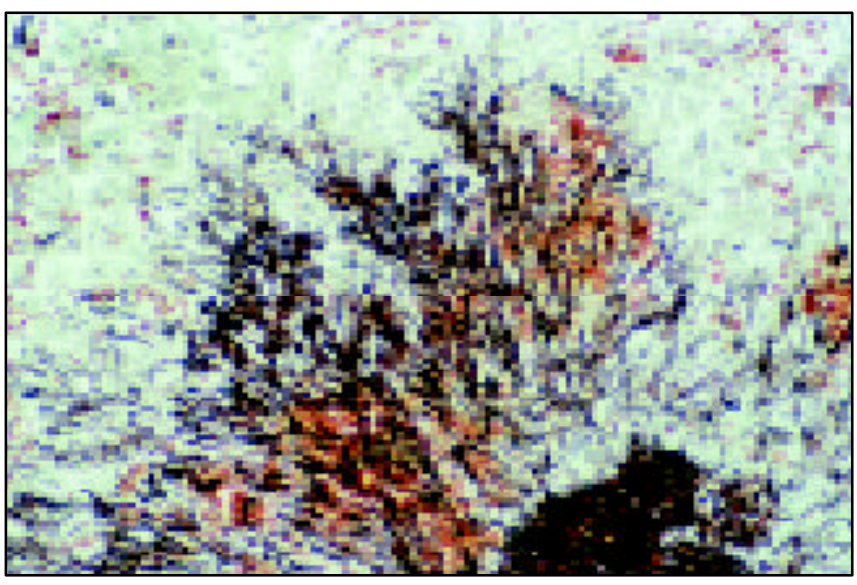

D 
no número de pacientes em risco de desenvolver a doença. Estas razões incluem o desenvolvimento de novos regimes intensivos de quimioterapia para tumores sólidos, linfomas de difícil tratamento, mieloma e leucemias resistentes, assim como um aumento no número de transplantes de órgãos sólidos e no uso de regimes imunossupressivos para outras doenças auto-imunes.
Apesar de poder ter uma contagem normal de neutrófilos, os pacientes afetados têm neutropenia funcional, porque a função dos neutrófilos está inibida pelo uso de altas doses de esteróides ${ }^{(7)}$. Por outro lado, a aspergilose invasiva é incomum em pacientes com a síndrome da imunodeficiência adquirida, provavelmente porque a deficiência imunológica destes pacientes afe- ta principalmente as células $\mathrm{T}$, mais do que os granulócitos e macrófagos ${ }^{(9)}$.

$\mathrm{Na}$ aspergilose invasiva os organismos podem invadir os vasos sanguíneos ou as vias aéreas. Na forma invasiva das vias aéreas o diagnóstico é feito pelo encontro do Aspergillus, profundamente, na membrana basal da via aérea envolvida. Isto diferencia da aspergilose broncopulmonar

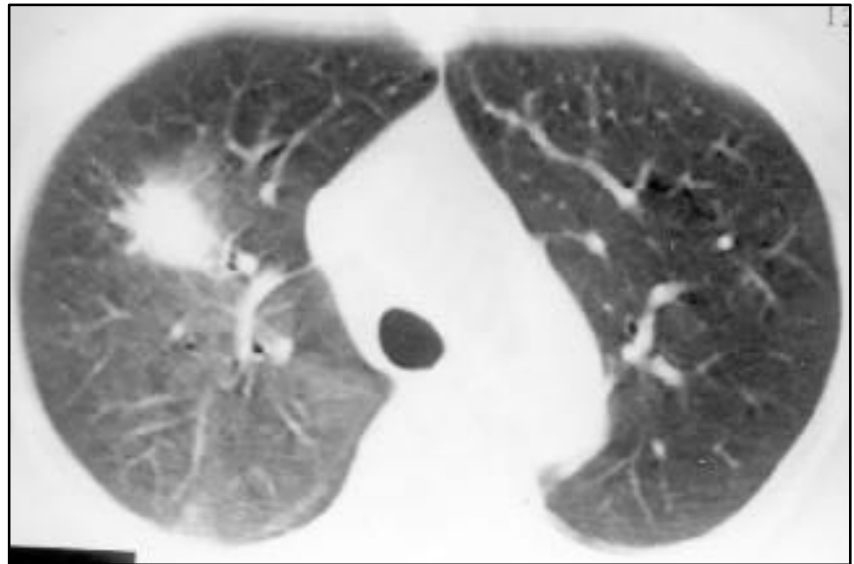

A

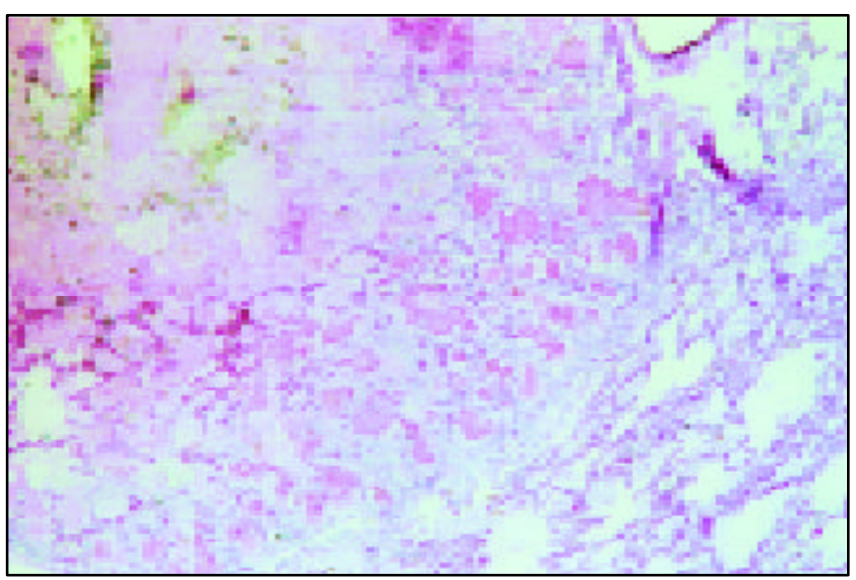

B

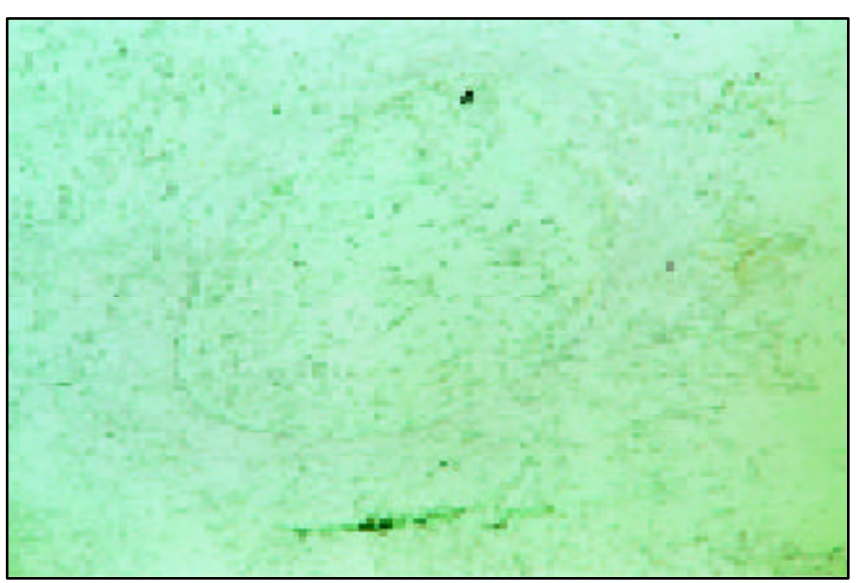

D
Figura 2. Em A, TCAR evidenciando nódulo no lobo superior direito, de contornos irregulares, com extensa área de atenuação em vidro fosco, não só ao redor do nódulo, mas ocupando grande parte do segmento posterior. Em $\mathbf{B}$, coloração pela hematoxilina-eosina, observa-se a interface do nódulo com o parênquima normal (direita). O nódulo (esquerda) corresponde a zona necro-hemorrágica, com espaços alveolares cheios de sangue na periferia. Em C, corte com coloração pela prata mostrando grande quantidade de hifas do fungo no interior do nódulo. Em $\mathbf{D}$ e E, colorações pelo PAS e pela prata, observam-se fungos no interior de arteríolas, uma delas parcialmente trombosada, caracterizando o aspecto de angioinvasão.

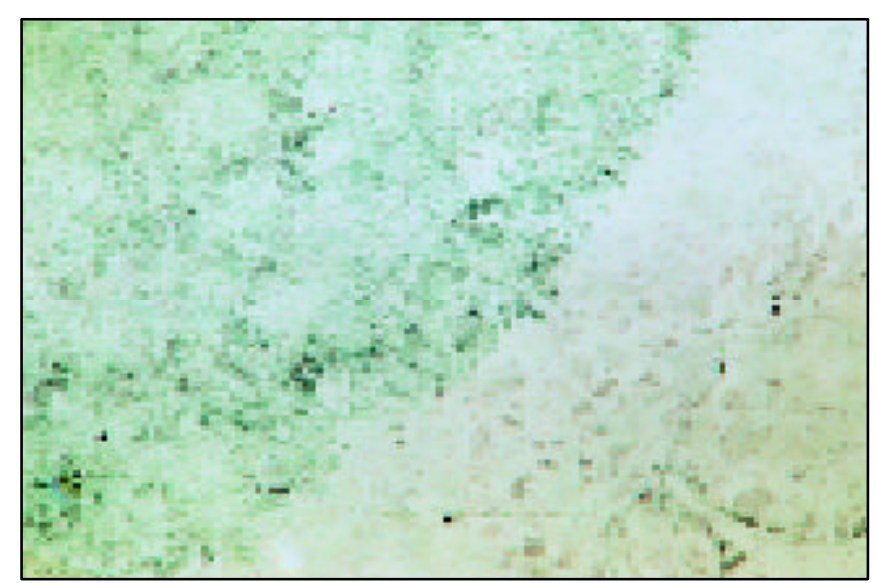

C

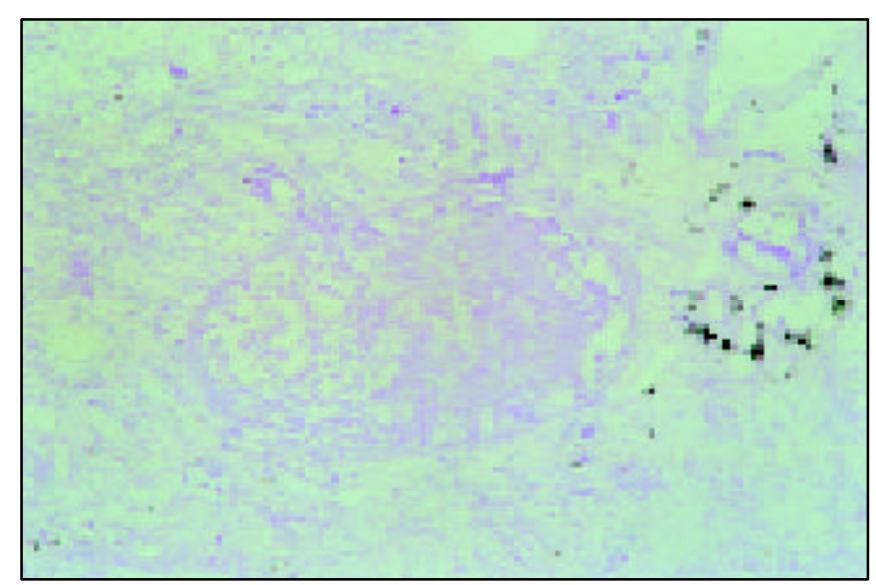

E 
alérgica, na qual os organismos ficam somente na luz brônquica ${ }^{(8)}$. Na TCAR, o quadro mais comum é o de áreas de consolidação peribrônquicas, ou de nódulos centrolobulares $^{(2)}$.

A manifestação mais comum da AAI são os infartos hemorrágicos com angioinvasão. Os achados característicos da AAI na TCAR consistem de nódulos envoltos por halo em vidro fosco (sinal do halo), ou áreas de consolidação segmentares, por vezes em forma de cunha, com a base voltada para a superfície pleural, associadas ou não a áreas de atenuação em vidro fosco $^{(2,7)}$. Estes achados correspondem a infartos hemorrágicos ${ }^{(7)}$.

Em pacientes com neutropenia grave, especialmente nos com leucemia aguda, o sinal do halo é altamente sugestivo de AAI ${ }^{(3,4,7)}$, embora em contextos clínicos diferentes possa ser observado em várias outras doenças ${ }^{(7,10)}$.

Com a evolução para a cura, o tecido necrótico central retrai e se afasta do tecido normal ao redor ${ }^{(\mathbf{1})}$. A separação de fragmentos de pulmão necrótico do parênquima adjacente resulta em crescentes aéreos similares aos vistos no micetoma. O sinal do crescente aéreo na AAI é visto durante a convalescença, isto é, duas a três semanas após o início do tratamento, e concomitante com a involução da neutropenia ${ }^{(7)}$.

Kuhlman et al. ${ }^{(\mathbf{3 , 4})}$ definiram bem as diferentes fases evolutivas do processo. $\mathrm{O}$ si- nal do halo é achado freqüente nas fases iniciais da doença, ocorrendo nos períodos de aplasia mais profunda da medula óssea, em geral três a quatro semanas após o início da quimioterapia. Uma ou duas semanas após, o processo escava e ocorre a formação do crescente aéreo. Estes achados surgem na fase de recuperação da medula óssea. Com a evolução, a AAI cicatriza com um padrão semelhante ao dos infartos pulmonares. As lesões gradativamente se tornam menores, por retração ou reabsorção da periferia, formando pequenos cistos, de paredes finas, com estrias lineares se estendendo para a superfície pleural.

A AAI caracteriza-se histologicamente por invasão e oclusão de artérias pulmonares de pequeno e médio calibres pelas hifas do fungo, associadas a lesão endotelial e trombose. Isto leva à formação de nódulos necróticos hemorrágicos, ou infartos hemorrágicos em forma de cunha, adjacentes à superfície pleural ${ }^{(\mathbf{7 , 8})}$. Estes nódulos são freqüentemente envoltos por áreas de hemorragia $^{(\mathbf{3})}$ e escasso infiltrado linfocitário. Assim, a manifestação mais comum da doença são os infartos hemorrágicos ${ }^{(2)}$.

O diagnóstico clínico é difícil e a mortalidade é muito elevada ${ }^{(7)}$. De maneira geral, o prognóstico é ruim e taxas de mortalidade entre $65 \%$ e $70 \%$ são relatadas ${ }^{(4)}$. Kuhlman et al. ${ }^{(4)}$ citam que o seu grupo tem conseguido sobrevida superior a $80 \%$, e atribuem este sucesso aos diagnósticos pre- coces feitos a partir do uso rotineiro da TCAR nesses pacientes.

\section{REFERÊNCIAS}

1. Aquino SL, Kee ST, Warnock ML, Gamsu G. Pulmonary aspergillosis: imaging findings with pathologic correlation. AJR 1994;163:811-5.

2. Won HJ, Lee KS, Cheon JE, et al. Invasive pulmonary aspergillosis: prediction at thin-section $\mathrm{CT}$ in patients with neutropenia - a prospective study. Radiology 1998;208:777-82.

3. Kuhlman JE, Fishman EK, Burch PA, Karp JE, Zerhouni EA, Siegelman SS. CT of invasive pulmonary aspergillosis. AJR 1988;150:1015-20.

4. Kuhlman JE, Fishman EK, Burch PA, Karp JE, Zerhouni EA, Siegelman SS. Invasive pulmonary aspergillosis in acute leukemia. The contribution of CT to early diagnosis and aggressive management. Chest 1987;92:95-9.

5. Franquet T, Muller NL, Gimenez A, Domingo P, Plaza V, Bordes R. Semiinvasive pulmonary aspergillosis in chronic obstructive pulmonary disease: radiologic and pathologic findings in nine patients. AJR 2000;174:51-6.

6. Kim SY, Lee KS, Han J, et al. Semiinvasive pulmonary aspergillosis: $\mathrm{CT}$ and pathologic findings in six patients. AJR 2000;174:795-8.

7. Franquet T, Muller NL, Gimenez A, Guembe P, Torre J, Bagué S. Spectrum of pulmonary aspergillosis: histologic, clinical, and radiologic findings. Radiographics 2001;21:825-37.

8. Logan PM, Primack SL, Miller RR, Muller NL. Invasive aspergillosis of the airways: radiographic, CT and pathologic findings. Radiology 1994; 193:383-8.

9. Staples CA, Kang EY, Wright JL, Phillips P, Muller NL. Invasive pulmonary aspergillosis in AIDS: radiographic, $\mathrm{CT}$ and pathologic findings. Radiology 1995;196:409-14.

10. Persegani MK, Marchiori E, Rodrigues R, et al. O "sinal do halo" na tomografia computadorizada de alta resolução do tórax. Rev Imagem 2001;23: 225-31. 